\title{
A Simple Optical System for Miniature Spindle Runout Monitoring
}

ChaBum Lee, Rui Zhao, and Seongkyul Jeon

Department of Mechanical Engineering, Tennessee Technological University, 1 William L Jones Dr., Cookeville, TN, 38505, USA

\begin{abstract}
We proposed a novel optical technique to monitor miniature spindle runout in a simple manner. Miniature spindles are commonly used in many machining applications, for example: micro-milling and micro-grinding. However, the capacitive sensors (CS) or eddy current sensors typically used for spindle runout measurements cannot be used for miniature spindle systems. This is due to the nonlinearity of the charge between a curved surface and a flat plate (sensing area) and a curved surface (measuring target area) and the effective sensing area being larger than the measuring target area. The proposed sensor utilizes curved-edge diffraction (CED), which uses the effect of the cylindrical surface curvature on the diffraction phenomenon in the transition regions adjacent to shadow, transmission, and reflection boundaries. The laser beam is incident to the spindle shaft edges along the $\mathrm{Y}$ and $\mathrm{Z}$ axes, four photodetectors then collect the total fields produced by the interference created by the waves due to CED around the spindle shaft edges. Two CS were used as a baseline comparison with the proposed sensor's performance. A spindle with a shaft diameter of $\phi 5.0 \mathrm{~mm}$ (same as CS effective sensing area) was selected to compare the results of the curved-edge sensor (CES) with the results of the CS. The spindle runout was measured and the following results were found: CES-CS calibration nonlinearity $(\mathrm{Z} 0.35 \%$ and $\mathrm{Y}$ $0.40 \%$ ) and resolution ( $\mathrm{Z} 20.1 \mathrm{~nm}$ and $\mathrm{Y} 26.0 \mathrm{~nm}$ for $\mathrm{CS}$ and Z $20.3 \mathrm{~nm}$ and Y $15.9 \mathrm{~nm}$ for CES). The fundamental sensing limit of CES was estimated to be: $Z 0.52 \mathrm{~nm} / \sqrt{\mathrm{Hz}}$ and $Y 0.41 \mathrm{~nm} / \sqrt{\mathrm{Hz}}$ for a working range of approximately $100 \mu \mathrm{m}$, respectively.
\end{abstract}

Keywords: Sensors, Spindle health monitoring, Spindle runout, Curved edge diffraction, Optical sensing

\section{Introduction}

Spindle runout is a rotation inaccuracy which occurs when the cutting tool is no longer aligned with the rotational axis, and can be classified as axial runout and radial runout. The former occurs when the cutting tool is rotating at an angle to the axis [1-6], and the latter occurs when the cutting tool is rotating off center to the rotational axis. Spindle runout in computer numerical control (CNC) machine tools can decrease tool life and leads to the machining of defective parts, for example, in drilling applications, spindle runout can result in a bore diameter that is actually larger than the drill's nominal diameter [6-8]. A spindle can be measured either dynamically or statically by various contact or non-contact sensing systems. The static measurement is significantly cheaper and easier, yet somewhat less accurate than dynamic measurement because it is unable to account for heat, vibration, and centrifugal forces acting on the spindle system. 
Capacitive sensors (CS) or eddy current sensors (ECS) are particularly well suited for measuring the performance of high speed precision spindles and other rotating shafts $[6,8]$. Since CS and ECS provide high measurement bandwidth and fast response, they are capable of gathering data even as the spindle or shaft sweeps through its entire speed range. However, it is standard practice for commercial CS and ECS to be factory calibrated with flat target surfaces and not curved target surfaces. As a general rule, the measuring target should also be 30 50\% larger than the CS in use (manufactures' recommendation) [911]. If the measuring target is not large enough to support the electric field it will tend to wrap around the target edge and enter normal to the target side. This electric field distortion will create measurement errors by degrading the sensor linearity and changing its sensitivity and measuring range. Due to the different behavior of the electric field between two interfacing surfaces when measuring a curved target surface, the error in sensitivity may be as much as $150 \%$. Furthermore, the nonlinearity may prevent accurate measurements with small diameter targets [12,13]. Measuring targets that are too small to support the electric field can also provide false displacement signals from lateral motions. Additionally, if a tilted or curved target is being measured the electric field will be distorted and the accuracy will be compromised because the CS or ECS will measure the average distance to the target under the area of the sensor and the measuring target surface will prevent the CS and CES from full target contact. Thus, there is no proper measuring devices for the high precision displacement measurement of curved, relatively small (smaller than the effective sensing area of CS) target surfaces.

Since these effects lead to critical measurement error in precision metrology for manufacturing applications requiring the highest accuracy, to fill the technology gap, it is urgent that a new rigorous sensing methodology for precision dimensional metrology of curved target surfaces be investigated. Lee recently introduced a displacement sensing technique utilizing curved edge diffraction (CED) for the first time [13]. However, only the experimental approach was considered because the theoretical approach requires complex mathematical and computational work to model. In this article, a novel optical sensing method that can overcome the current issues with CS and ECS for the miniature spindle metrology has been discussed using theoretical and experimental approaches.

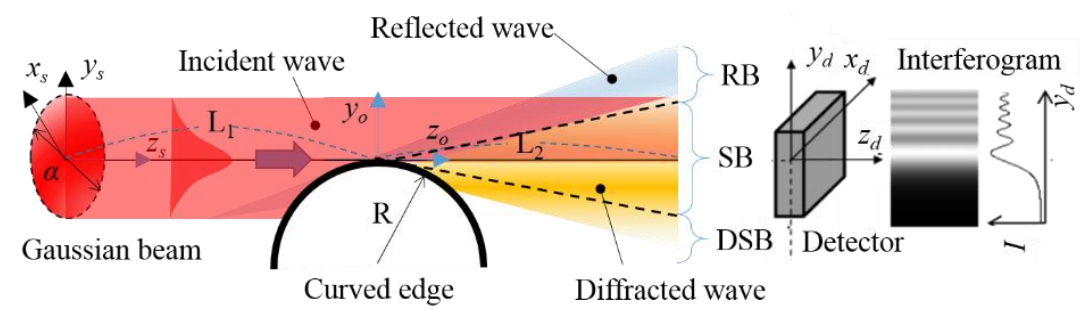

FIG. 1. Measurement principle of CES: $x_{i}$ and $y_{i}$ is an $i$ coordinate (source, spindle, or detector denoted as s, o, and $\mathrm{d}$, respectively) system at $z=z_{i}$, respectively.

\section{Measurement Principle}

The knife-edge sensing techniques for dimensional measurement applications have been recently introduced [1417]. In particular, Lee proposed a new linear displacement sensing method utilizing knife-edge diffraction (KED) $[16,17]$. An electromagnetic (EM) wave incident on the knife-edge gives rise to two waves, a transmitted wave and an edge-diffracted wave. The total electric field represented by a sum of the two waves creates an interferogram at the detector plane. A high sensor sensitivity can be observed near the center of a Gaussian laser 
beam. The convenient and efficient computational method for KED problems in the transition region adjacent to the shadow boundary was introduced. However, unlike KED, an analytical approach to CED is difficult because CED can be understood from the asymptotic solution of several canonical problems, which involve the illumination of the edge by diffraction wavefronts [18]. In this work, CED solutions were formulated in a compact, accurate form using the diffraction characteristics valid in the transition region adjacent to the shadow and reflection boundaries. As shown in FIG. 1, an electromagnetic EM wave incident on the curved edge, with radius of curvature: $R$, gives rise to an incident wave, diffracted wave, and reflected wave, which propagates along a surface ray. RB, SB and DSB represent reflection boundary, shadow boundary, and deep shadow boundary, respectively. Such surface ray fields may also be excited at the transition region and can be separated into three different regions similar to KED. The total electric field may be represented by a sum of three waves, and can be observed at the detector plane. This sum predicts the scattering of the optical large platform that involves the use of spatial domain Fresnel integrals for the diffracted fields. Assuming that the light source with a Gaussian intensity profile is not diverging, the detector size is relatively small compared to the distance $L_{2}$ between the curved edge and the detector, and the detector is placed along the light propagation axis, only the transmitted wave and edge-diffracted wave can be considered. Two waves are superimposed to generate an interference fringe at the detector plane, this contributes to an increase in the peak power of the $1^{\text {st }}$ fringe. The steep slope of this signal plays a role in increasing the sensor sensitivity up to approximately $40 \%$ due to the edge diffraction principle $[16,19,20]$.

The incident field $\vec{E}_{s}$ with a Gaussian aperture distribution can be defined as [21,22]

$$
\vec{E}_{s}\left(x_{s}, y_{s}, z_{s}\right)=E_{0} e^{-j k_{0} z_{s}} e^{-\frac{x_{s}^{2}+y_{s}^{2}}{\alpha^{2}}}
$$

Where $E_{0}$ is the amplitude of the incident field, $k_{0}$ is the wavenumber in air, and $\alpha$ is the beam width. The incident field $\vec{E}_{s}$ creates constructive and destructive interferences with the diffracted field created by a phase change at the knife-edge. The superimposed wave can be obtained by applying a Fourier transform (FT), and the total field $\vec{E}_{d}$ (sum of incident and diffracted fields) at the detector, can be obtained by taking the inverse FT of the superimposed wave $[16,19,20]$. The total field measured along the $z$ axis can be expressed as

$$
\vec{E}_{d}\left(x_{d}, y_{d}, z_{d}\right)=\frac{1}{(2 \pi)^{2}} \iint_{k-\text { space }} e^{-j \vec{k} \cdot \vec{r}_{0 \rightarrow d}}\left[\iint_{\text {aperture }} \vec{E}_{s} e^{j \vec{k} \cdot \vec{r}_{s \rightarrow 0}} d x d y\right] d k_{x} d k_{y}
$$

Where $\vec{r}_{i \rightarrow j}$ is a position vector from $i$ to $j$ in the coordinate system.

Finally, the power of the combined fields at a detector can be calculated as

$$
P_{j}\left(y_{o}\right)=\iint_{m \times m} \vec{E}_{d}\left(x_{d}, y_{d}, z_{i}=L_{2}\right) \cdot\left\langle\vec{E}_{d}^{*}\left(x_{d}, y_{d}, z_{i}=L_{2}\right)\right\rangle^{*} d x d y .
$$


where \langle\rangle$^{*}$ denotes a complex conjugate of $\vec{E}_{d}\left(x_{d}, y_{d}, z_{i}=L_{2}\right)$, and the total power $P_{j}$ induced by CED at the detector will be calculated by multiplying the total field and the conjugated total field at each detector plane and integrating over the detector size $(m \times m)$. The edge diffraction patterns can be obtained from Eq. (3).

As shown in FIG. 2, the edge diffraction fringe is clearly observed under the conditions: $L_{2}=100 \mathrm{~mm}, \lambda=633 \mathrm{~nm}$, $\alpha=0.5 \mathrm{~mm}, m=0 \mathrm{~mm}$ (point source), while the fringe is not clearly seen in the case of $m=0.5 \mathrm{~mm}$. This result indicates that the detector size has a significant influence on the CED fringe pattern, especially the first peak. In addition to the detector size effect, the surface roughness of the spindle may also have an effect on the CED fringe pattern, and some portion of the scattering field exists because of the surface roughness because the diffracted field becomes incoherent and the interference fringe becomes weak when the light is incident on the rough surface. In this work, the surface roughness effect has not been considered, and dimensional sensing characteristics from the spindle shaft surface has been the focus.

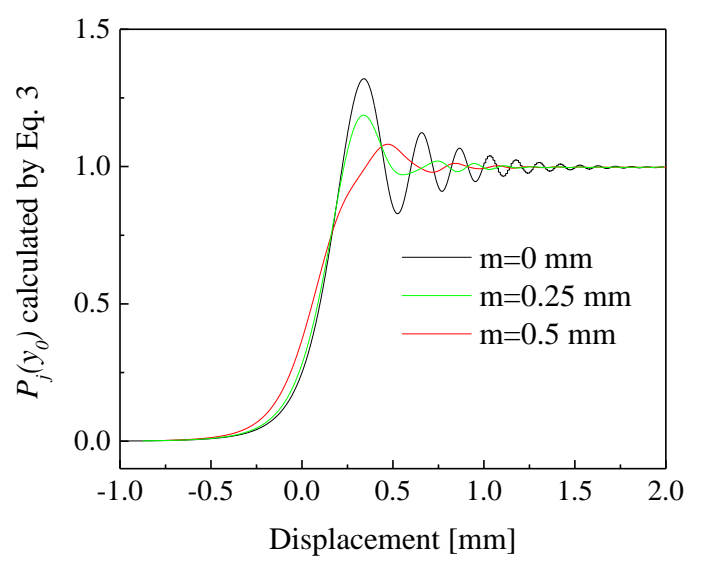

FIG. 2. Total power of the combined fields calculated by Eq. (3) with respect to the detector size $(m \times m)$.

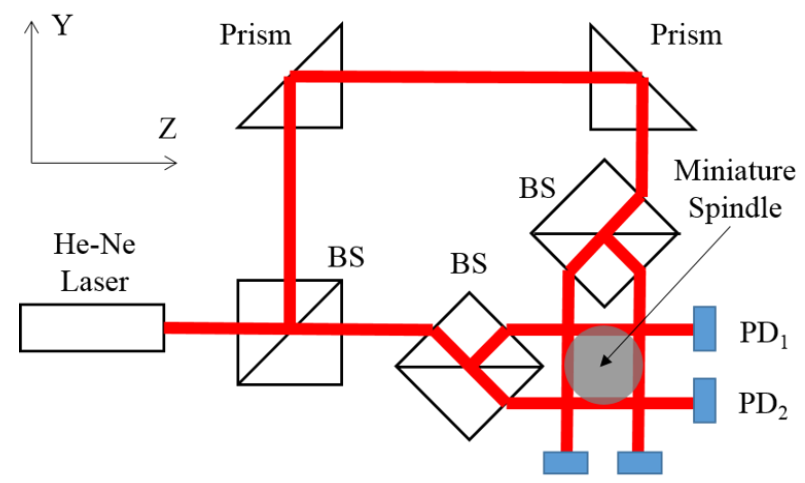

$\begin{array}{ll}\mathrm{PD}_{4} & \mathrm{PD}_{3}\end{array}$

FIG. 3. Schematic illustration of the spindle runout measurement.

\section{Experiments}


In the experiment, as illustrated in FIG. 3, the laser beam $(\lambda 633 \mathrm{~nm}$, power $2.3 \mathrm{~mW}, \alpha 0.5 \mathrm{~mm})$ is split into four by using beam splitters (BS), and each laser beam is incident on the cylindrical edge surface of a precision-level ball bearing spindle system (10,000 rpm max.) with a 90 degree interval. The artifact (shaft diameter $\phi 5.0 \mathrm{~mm})$ was prepared and bolt-clamped with a spindle head. Four Si photodetectors (PD, effective area $0.25 \mathrm{~mm}^{2}$ ) were placed $100 \mathrm{~mm}$ away from the spindle system, the distances $\left(\mathrm{PD}_{1}\right.$-to- $\mathrm{PD}_{2}, \mathrm{PD}_{3}$-to- $\left.\mathrm{PD}_{4}\right)$ were placed the same distance apart as the spindle shaft diameter of $\phi 5 \mathrm{~mm}$. The PD array collects the total power of each beam in a differential configuration [3,14,15], which eliminates the dependency on the laser intensity. Low pass filters with a cut-off frequency of $1.5 \mathrm{kHz}$ (measurement bandwidth) were added at the end of the four PD outputs. Consequently, the sensor outputs, $V_{\text {out }}(Y)$ and $V_{\text {out }}(Z)$, can be expressed as,

$$
V_{\text {out }}(Y)=\frac{P_{2}\left(y_{o}\right)-P_{1}\left(y_{o}\right)}{P_{2}\left(y_{o}\right)+P_{1}\left(y_{o}\right)}, V_{\text {out }}(Z)=\frac{P_{4}\left(z_{o}\right)-P_{3}\left(z_{o}\right)}{P_{4}\left(z_{o}\right)+P_{3}\left(z_{o}\right)} .
$$
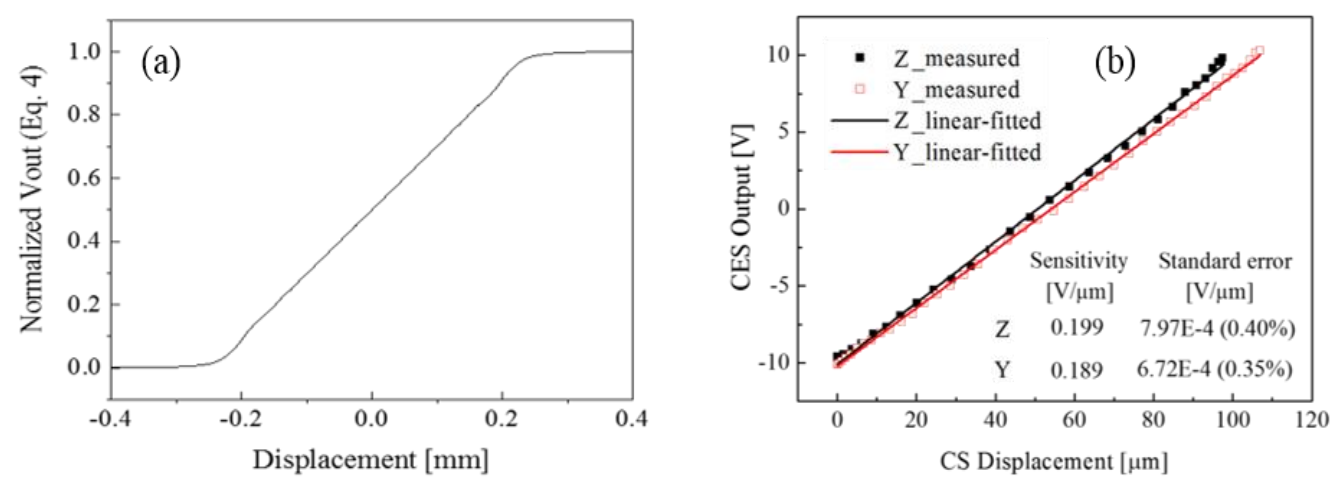

FIG. 4. Sensor output results: (a) theoretical calculation by Eq. 4 and (b) calibration results.

The proposed curved-edge sensor (CES) outputs were collected with an NI LabView data acquisition system, and were calibrated using the CS as reference (10 nm resolution, effective sensing area $\phi 5.0 \mathrm{~mm}$ ) within approximately $100 \mu \mathrm{m}$ measuring range by moving the spindle shaft along the $\mathrm{Y}$ and $\mathrm{Z}$ axes, respectively. Because two CS cannot be installed around a $\phi 5.0 \mathrm{~mm}$ spindle shaft at the same time due to mechanical contact between the two CS in the limited space, the CES was calibrated axis by axis along the $\mathrm{Y}$ and $\mathrm{Z}$ axes, respectively. As shown in FIG. 4, the CES outputs showed a high

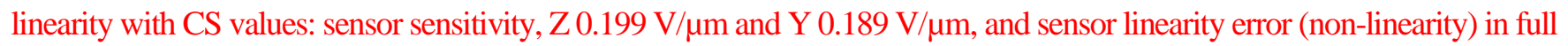
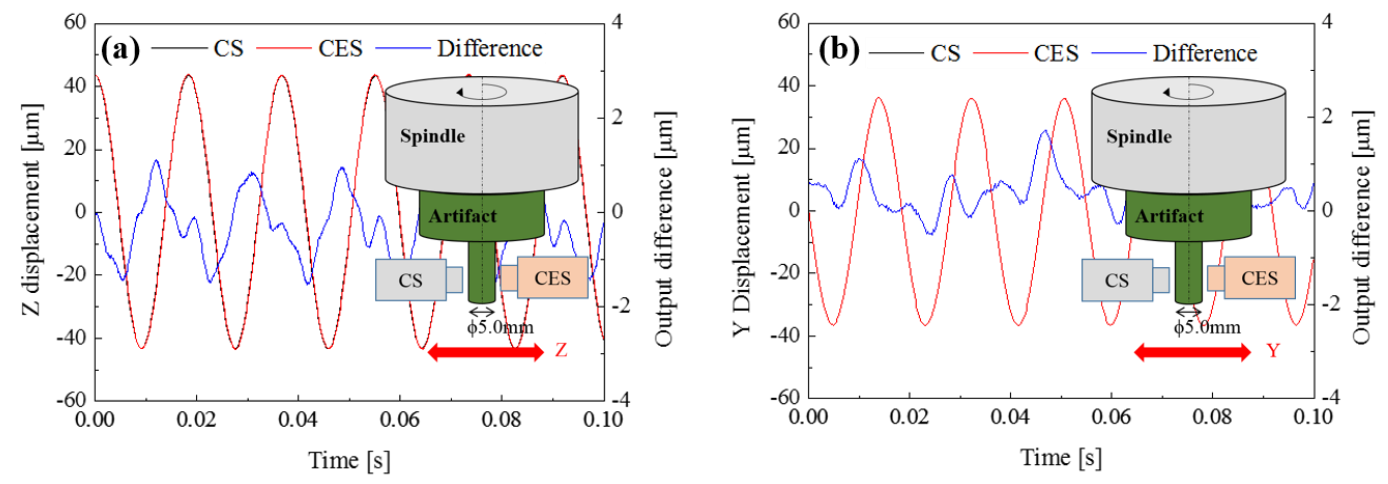

FIG. 5. Comparison of CS and CES outputs at $3200 \mathrm{rpm}$ according to the (a) $\mathrm{Z}$ and (b) $\mathrm{Y}$ axes. 
scale, Z $0.4 \%$ and Y $0.35 \%$, were obtained. Sensor linearity error was calculated as a sum of the residual error in leastsquares fitting of a linear fitted curve. Also, the sensor output curve estimated by Eq. (4) was found linear within a $\pm 0.1 \mathrm{~mm}$ range. Also, the CES measured the spindle runout at $3200 \mathrm{rpm}$ (revolution per min.) along the $\mathrm{Y}$ and $\mathrm{Z}$ axes, respectively, as shown in FIG. 5. Here, the spindle runout includes spindle motion error at a given frequency and the roundness error of the artifact. The discrepancy between CS and CES was approximately Z $2.5 \mu \mathrm{m}$ and Y $2.0 \mu \mathrm{m}$. This discrepancy was thought to be due to sensor characteristics and sensor alignment errors. Also, two sensor outputs were monitored for 20 min. along the $Y$ and Z axes to assess CES reliability (FIG. 6): Z $20.1 \mathrm{~nm}$ and Y $26.0 \mathrm{~nm}$ for CS and Z $20.3 \mathrm{~nm}$ and Y $15.9 \mathrm{~nm}$ for CES. It was found that the CES reliability was similar to the CS reliability. Here, the root-mean-square (rms) noise of sensor signals was used to determine the sensing resolution.
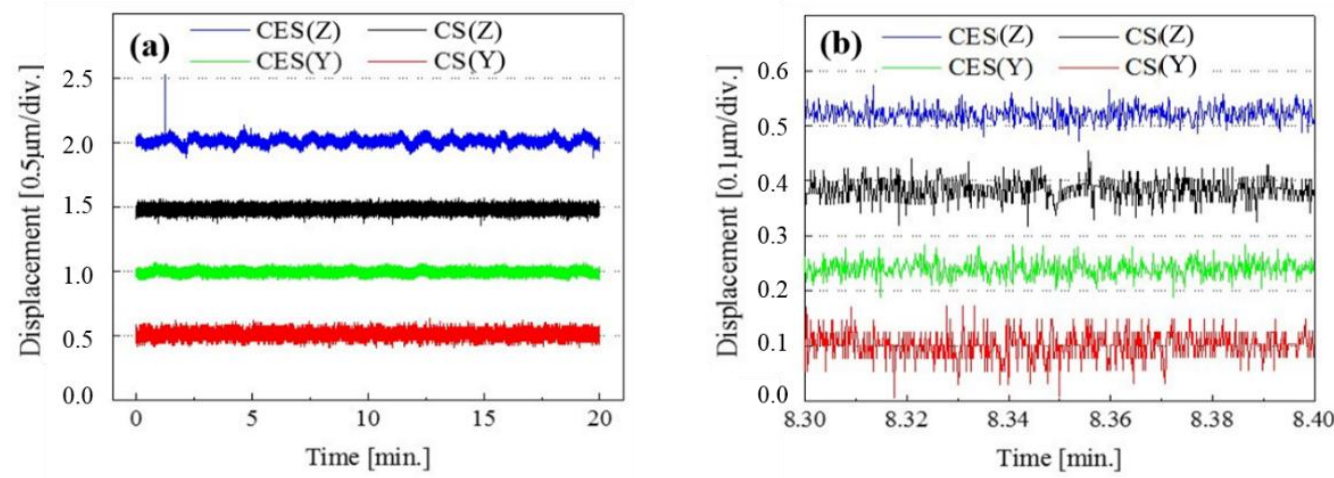

FIG. 6. Stability comparison of CS and CES: (a) 20min. stability test and (b) details.

The fundamental sensing limit of CES in the present experiment setup can be determined by the photonic shot noise $\left(N_{S}\right)$ in Eq. (1) $[14,22,23]$,

$$
N_{s}=\frac{\Delta R}{\sqrt{\Delta f}}
$$

Where, $\Delta f$ and $\Delta R$ are the measurement bandwidth and resolution, respectively. The photonic shot noise indicates the variation of the sensing signal as a function of its resolution and measurement bandwidth. Thus, the fundamental sensing limit of CES was estimated: $Z 0.52 \mathrm{~nm} / \sqrt{\mathrm{Hz}}$ and $Y 0.41 \mathrm{~nm} / \sqrt{\mathrm{Hz}}$ for working ranges of approximately $100 \mu \mathrm{m}$. This noise can be reduced by using a sufficiently bright light source with a large beam diameter relative to the PD $[3,24,25]$. 


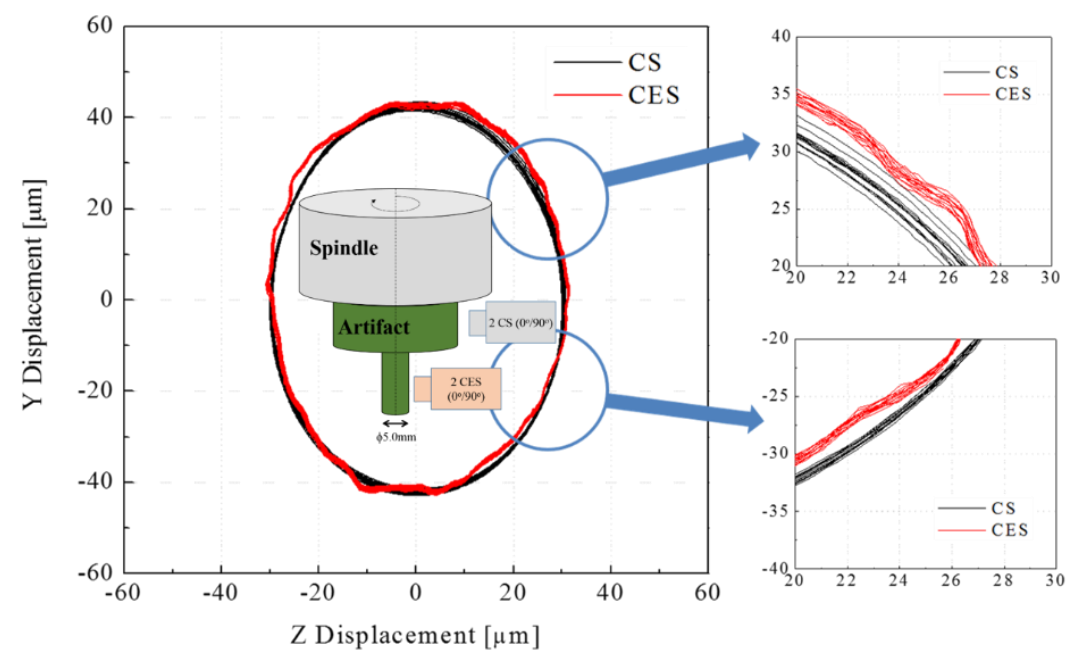

FIG. 7. Spindle runout measurement at 8600 rpm: approximately $\mathrm{Z} \pm 30 \mu \mathrm{m}$ and $\mathrm{Y} \pm 42 \mu \mathrm{m}$.

The spindle runout was simultaneously measured with both the CS and the CES. As illustrated in FIG. 7, two CS were placed around the flange $(\phi 40 \mathrm{~mm})$ of the artifact with a 90 degree interval, because two CS cannot be installed around the artifact nose $(\phi 5.0 \mathrm{~mm})$ at the same time as the size of the CS makes it impossible. Two CES were placed around the artifact nose. Two sensors were orthogonally placed around the spindle shaft along the $\mathrm{Y}$ and $\mathrm{Z}$ axes. While the spindle was rotating at $8600 \mathrm{rpm}$, the spindle runout, including spindle motion errors, and the roundness error of the artifact was measured with CES. These results were compared to CS outputs along the $\mathrm{Y}$ and $\mathrm{Z}$ axes. Because the CS, with its effective sensing area of $\phi$ $5.0 \mathrm{~mm}$, measures the curved surface of the artifact, it is limited to the spindle motion measurement with high spatial and temporal frequency. Such CS typically average the target's surface properties (roughness, roundness). Thus, the effective sensing area of the CS has to be smaller (at least 10 times, according to CS manufacturers' recommendation) than the spindle shaft diameter to effectively measure the spindle motions, but a CS with a small $(\phi 0.5 \mathrm{~mm})$ effective sensing area is limited to a few tens of micrometer measuring range. On the other hand, the beam diameter of the laser source used in the experiment is $\phi 0.5 \mathrm{~mm}$, and the proposed CES has a measuring range of approximately $100 \mu \mathrm{m}$. As shown in FIG. 7, two sensors showed a similar spindle runout: approximately $\mathrm{Z} \pm 30 \mu \mathrm{m}$ and $\mathrm{Y} \pm 42 \mu \mathrm{m}$, and CES measurement results were repeatable although the CES output signal was prone to wobbling. These results indicate that CES can measure the miniature spindle motions with higher resolution when compared to the CS. Unlike the CS measurements, which average the spindle target surface and are sensitive to parasitic or lateral motions (such as $\mathrm{Z}$ motion along the $\mathrm{Y}$ axis and vice versa), CES measurement results could represent the true spindle runout. A direct comparison between two sensor outputs is not possible in this work because it is difficult to find ideal sensors for spindle metrology. These reference sensors should be highly accurate, precise, and able to measure from a long range. Ideally they would also be lateral motion-insensitive and compact. It is for this reason why CS have been considered in this work as representative spindle metrology tools.

The ball bearing spindle system used in this research is a precision level, but the manufacturing tolerance of a spindle shaft machined from a local machining service agency is approximately $40 \mu \mathrm{m}$, which was estimated by measuring the diameter of the spindle shaft 12 times at 15 degree interval. It is a critical issue to separate the spindle error motions (roundness, roughness, unbalance motion) independently. For the next work, the spindle 
error separation research with air spindle systems will be studied by CES, and additional validation can be experimentally done with another reference sensor with a high resolution and small effective measuring area.

\section{Conclusion}

We created a design principle for CES by deriving a mathematical model, characterized CES performance in terms of working range, linearity, and stability, and applied CES to spindle metrology. It was found that the proposed sensing technique is advantageous in achieving both high linearity (nonlinearity $0.35 \%$ ) and resolution $(15.9 \mathrm{~nm})$ within a $100 \mu \mathrm{m}$ full scale measuring range and in effectively measuring the miniature spindle motion in a simple and convenient manner. The bandwidth of the sensor is limited by the bandwidth of the electronics because the CES includes PDs, but even with these limitations the CES can still reach MHz levels of bandwidth depending on the filtering operation used. It is anticipated that the proposed sensor can be applied to in-situ spindle health monitoring applications, or act as an alternative to CS and perhaps be considered a standard selection in spindle metrology.

\section{Acknowledgements}

The research was supported by NSF (Award Number: CMMI 1463502) through Tennessee Technological University. Similarly, this work was supported by the Center for Manufacturing Research at Tennessee Technological University.

\section{References}

1. W. Gao, Precision nanometrology: Sensors and measuring systems for nanomanufacturing, Springer (2010).

2. K. Liu, M. Sun, T. Zhu, Y. Wu, Y. Liu, Modeling and compensation for spindle's radial thermal drift error on a vertical machining center, Int. J. Mach. Tools Manuf., 105, 58-67 (2016).

3. T. Oiwa, K. Kaneko and A. Kyusojin, Motion error compensation of a mechanical contact spindle using a multi-degreeof-freedom micropositioning stage, Rev. Sci. Instrum. 76, 025104 (2005).

4. M. Madden, M. Aketagawa, S. Uesugi, T. Kumagai and E. Okuyama, Spindle error motion measurement using concentric circle grating and phase modulation interferometer, Int. J. Automation Technol., 7(5), 506-513 (2013).

5. Y. Hwang, G-H. Kim, Y-B. Kim, J-H. Kim, S-K. Lee, Suppression of the inflection pattern in ultraprecision grinding through the minimization of the hydrodynamic force using a toothed wheel, Int. J. Mach. Tools Manuf., 100, 105-115 (2015).

6. M. Madden, M. Aketagawa, T. Kumagai, Y. Maeda and E. Okuyama, Concurrent measurement method of spindle radial, axial and angular motions using concentric circle grating and phase modulation interferometers, Meas. Sci. Technol. 25, 094005 (2014).

7. J. Kim, S. Zhao, G. Kim and S-K. Lee, Rolling bearing-suspended spindle run-out control using repetitive control and adaptive feedforward cancelation, Int. J. Prec. Eng. Manuf., 14(12), 2171-2178 (2013).

8. J. Kim and S-K. Lee, Micro-patterning technique using a rotating cutting tool controlled by an electromagnetic actuator, Int. J. Mach. Tools Manuf., 101, 52-64 (2016). 


\section{9. http://www.mtiinstruments.com.}

10. http://www.lionprecision.com.

11. http://www.capacitance-sensors.com.

12. P. T. Smith, R. R. Vallance and E. R. Marsh, Correcting capacitive displacement measurements in metrology applications with cylindrical artifacts, Precision Engineering, 29(3), 324-335 (2005).

13. C. Lee, S. M. Mahajan, R. Zhao, and S Jeon, A curved edge diffraction-utilized displacement sensor for spindle metrology, Rev. Sci. Instrum. 87, 075113 (2016).

14. C. Braunsmann, V. Prucker and T. E. Schäffer, Optical knife-edge displacement sensor for high-speed atomic force microscopy, Appl. Phys. Lett. 104, 103101 (2014).

15. D. Karabacak, T. Kouh, C. C. Huang and K. L., Optical knife-edge technique for nanomechanical displacement detection, Appl. Phys. Lett. 88, 193122 (2006).

16. C. Lee, S-K. Lee and J. A. Tarbutton, Novel design and sensitivity analysis of displacement measurement system utilizing knife edge diffraction for nanopositioning stages, Rev. Sci. Instrum. 85, 095113 (2014).

17. C. Lee, J. A. Tarbutton and S-K. Lee, Positioning control effectiveness of optical knife edge displacement sensorembedded monolithic precision stage, Sen. Actuators A, 233, 390-396 (2015).

18. G. L. James, Geometrical Theory of Diffraction for Electromagnetic Waves, $3^{\text {rd }}$ Ed., IET (1979).

19. C. L. Giovaneli, An analysis of simplified solutions for multiple knife-edge diffraction, IEEE Trans. Antennas Propag. 32(3), 297-301 (1984).

20. B. A. David and G. S. Brown, Diffraction by a randomly rough knife edge, IEEE Trans. Antennas Propag. 50(12), 17691788 (2012).

21. E. Hecht, Optics, $4^{\text {th }}$ Ed., Addison-Wesley (2001).

22. J. W. Goodman, Introduction to Fourier Optics, $3^{\text {rd }}$ Ed., Roberts and Company Publishers (2004).

23. T. E. Schäffer, Force spectroscopy with a large dynamic range using small cantilevers and an array detector, J. Appl. Phys. 91(7), 4739-4746 (2002).

24. C. Lee, G-H. Kim and S-K. Lee, Design and construction of a single unit multi-functional optical encoder for a sixdegree-of-freedom motion error measurement in an ultraprecision linear stage, Meas. Sci. Techno. 22, 105901 (2011).

25. C. Lee, G-H. Kim and S-K. Lee, Uncertainty Investigation of Grating Interferometry in Six-degree-of-freedom Motion Error Measurements, Int. J. Precis. Eng. Manuf. 13(9), 1509-1515 (2012). 